\title{
Flexible social organization and high incidence of drifting in the sweat bee, Halictus scabiosae
}

\author{
YUKO ULRICH, NICOLAS PERRIN and MICHEL CHAPUISAT \\ Department of Ecology and Evolution, University of Lausanne, Biophore, UNIL-Sorge, 1015 Lausanne, Switzerland
}

\begin{abstract}
The very diverse social systems of sweat bees make them interesting models to study social evolution. Here we focus on the dispersal behaviour and social organization of Halictus scabiosae, a common yet poorly known species of Europe. By combining field observations and genetic data, we show that females have multiple reproductive strategies, which generates a large diversity in the social structure of nests. A detailed microsatellite analysis of 60 nests revealed that $55 \%$ of the nests contained the offspring of a single female, whereas the rest had more complex social structures, with three clear cases of multiple females reproducing in the same nest and frequent occurrence of unrelated individuals. Drifting among nests was surprisingly common, as $16 \%$ of the 122 nests in the overall sample and $44 \%$ of the nests with complex social structure contained females that had genotypes consistent with being full-sisters of females sampled in other nests of the population. Drifters originated from nests with an above-average productivity and were unrelated to their nestmates, suggesting that drifting might be a strategy to avoid competition among related females. The sex-specific comparison of genetic differentiation indicated that dispersal was male-biased, which would reinforce local resource competition among females. The pattern of genetic differentiation among populations was consistent with a dynamic process of patch colonization and extinction, as expected from the unstable, anthropogenic habitat of this species. Overall, our data show that $H$. scabiosae varies greatly in dispersal behaviour and social organization. The surprisingly high frequency of drifters echoes recent findings in wasps and bees, calling for further investigation of the adaptive basis of drifting in the social insects.
\end{abstract}

Keywords: drifting, Halictidae, sex-biased dispersal, social organization, sweat bees

Received 19 November 2008; revision received 29 January 2009; accepted 5 February 2009

\section{Introduction}

Unravelling the causes of sociality, that is, why some species live in groups and engage in cooperative behaviour while many others are solitary, is a major challenge for evolutionary theory. Advanced eusocial species such as ants and termites provide limited insight into the early stages of social evolution, because in those species the females displaying altruistic behaviour are morphologically predisposed to this role. To understand the forces that promote sociality, it is therefore of particular interest to study species in which the social roles are flexible and individuals have the potential to behave altruistically or to reproduce, such as cooperatively breeding vertebrates and social insect species with totipotent individuals

Correspondence: Michel Chapuisat, Fax: +41 2169241 65; E-mail: michel.chapuisat@unil.ch
(Clutton-Brock 2002; Griffin \& West 2003; Hart \& Ratnieks 2005; Beekman et al. 2006).

The sweat bees (Hymenoptera, Halictidae) form one of the most labile taxa with respect to social organization. In this group, eusociality has arisen independently three times in a relatively recent time period (20-22 million years ago), with frequent subsequent reversals from eusocial to solitary behaviour (Danforth 2002; Brady et al. 2006). The social structure of sweat bees varies greatly across species, from strictly solitary to obligately eusocial. Variation can also be found within species, where social structure seems to correlate with environmental conditions, solitary nesting being often associated with harsher conditions, for example at high latitude (Richards 2000) or altitude (Eickwort et al. 1996). Social polymorphism is even found at the population level: in Halictus sexcinctus, a single population contained 'communal' nests composed of multiple adult females that reproduced more or less equally and eusocial nests composed of queens and 
their nonreproducing worker daughters (Richards et al. 2003). Similarly, in the first summer brood of Halictus rubicundus, some females stay and work in the natal nest while many others mate, diapause and found their own nest the following year (Yanega 1988). Because of this variation in cooperative nesting behaviour and social organization, halictine bees are commonly viewed as ideal model systems to study the conditions favouring the evolution of reproductive altruism and sociality (Packer \& Owen 1994; Schwarz et al. 2007).

Halictus scabiosae is a common ground-nesting halictine bee of Central and southern Europe. Nests are generally clustered on well-delimited patches of suitable habitat, mainly in anthropogenic sites or recently disturbed areas. The life cycle is annual and begins in spring when mated females emerge from hibernation and start provisioning their nests. Two broods are produced during summer. The first one is almost exclusively composed of females, an unknown proportion of which act as nonreproducing helpers in their natal nest. The second brood is composed of slightly larger females and males in similar proportions (Plateaux-Quénu 1972).

The social organization of $H$. scabiosae is poorly known, but earlier observations of very few nests suggest that there might be some variation in social structure over time and geographical location (Batra 1966; Knerer \& Plateaux-Quénu 1967). Several females often jointly founded nests in a population located in southwestern France (Knerer \& PlateauxQuénu 1966, 1967). One foundress became the dominant egg-layer, while the other subordinate females turned into foraging helpers. The main foundress gradually chased away her helpers, so that by the emergence of the first brood of females in summer, the nest had apparently become monogynous (i.e. with a single queen). In contrast, no clear relationship between behaviour, size and reproductive status was found when examining individuals sampled in summer from seven nests in a population near Geneva in Switzerland (Batra 1966). Moreover, some nests contained more than one individual with active ovaries and foragers were often mated, suggesting a polygynous social structure. These old observations suggest some interesting variation in reproductive behaviour, which calls for a molecular analysis of the genetic structure of nests to clarify the social organization and reproductive roles in this species (Ross 2001).

In this study, we investigated the life cycle, breeding system and dispersal of $H$. scabiosae. First, we observed the dynamics of nest founding and brood emergence in the field. Second, we used microsatellite markers to investigate the social organization of nests, paying special attention to the number of breeders, genetic relationship between these breeders and presence of unrelated, nonreproducing drifters. Third, we studied the genetic structure of the populations and inferred patterns of sex-specific dispersal. These data will further our understanding of the evolution of flexible reproductive and dispersal strategies in halictine bees.

\section{Materials and methods}

\section{Field monitoring and sampling}

We studied eight Halictus scabiosae populations located around Lausanne University, Switzerland, during spring and summer 2006 (Fig. S1 and Table S1, Supporting information). All populations were situated in anthropogenic habitat, including a paved path, slopes of mounds adjoining parking areas, a construction site and a public garden flowerbed. The distance between populations ranged between $200 \mathrm{~m}$ and $4.5 \mathrm{~km}$ (Fig. S1). The distance between nests within populations ranged between $3 \mathrm{~cm}$ and $21 \mathrm{~m}$. Population positions were determined with a global positioning system (GPS, Garmin, precision $\pm 2 \mathrm{~m}$ ). Nests within populations were precisely mapped with a metric tape. Nests were marked with numbered nails in the field.

To study the dynamics of nest founding, we monitored one population between 4 May and 15 August 2006. This population consisted of 42 nests clustered in a well-delimited area of approximately $2 \times 2 \mathrm{~m}$. Bees were collected at nest entrance with cone-shaped, net covered emergence traps. All females were marked with a three-colour code on the thorax, using a queen holder and enamel paint from a professional beekeeper marking kit. Similar marking had no perceptible effect on survival or behaviour in other species of the same genus (Yanega 1988, 1989; Richards et al. 2003). We recorded nest visits by individual females for 1 to $2 \mathrm{~h}$ per day over 38 days, usually in the morning when foraging activity was high.

To investigate the genetic structure and social organization of nests, we sampled 424 females and 58 males from eight populations for genetic and morphometric analyses (Tables S1 and S3, Supporting information). All individuals were captured at nest entrance between 25 July and 19 September, except seven females and one male that were caught during flight. Among the 122 sampled nests, there were 52 nests in which only one female was captured and 70 in which two or more females were captured. In 10 nests of the latter category, the time interval between the sampling of unrelated bees was greater than 12 days, so that the captured individuals may have occupied the nest successively, rather than together. To avoid incorrectly inferring polygyny in such cases, these 10 nests were excluded from the analyses of nestmate relatedness. In comparison, the mean ( \pm standard deviation) time interval between successive captures was 3.01 ( \pm 5.7 ) days for the 60 nests in which multiple females were captured and which were included in the analysis of nestmate relatedness.

Females were dissected and their degree of ovary activation was scored as: 0 (both ovaries thin and containing no developed oocytes), 0.5 (one ovary thick on less than half of its full length), 1 (one ovary thick on its full length or two ovaries thick on less than half of their length), 1.5 (one ovary 
thick on its full length, the other on less than half of its length) or 2 (both ovaries thick on their full length).

\section{DNA isolation and microsatellite analysis}

DNA was extracted from three crushed legs of each individual in $250 \mu \mathrm{L}$ of $5 \%$ Chelex (Sigma-Aldrich) and heated at $90{ }^{\circ} \mathrm{C}$ for $20 \mathrm{~min}$ (Chapuisat et al. 2004). We tested 38 microsatellite primer pairs coming from three halictid bee species: LHMS1, LHMS2, LHMS3, LHMS4, LHMS5, LHMS6, LHMS10, LHMS14, LHMS17 and LHMS18 that were developed for Lasioglossum hemichalceum (Kukuk et al. 2002), CT9, CT12, CT20, CT23 and CT24 that we designed from microsatellite sequences of L. hemichalceum available in GenBank (GenBank Accession nos AY823979-AY823983), mala01 mala04, mala06, mala07, mala09, mala10, LM11, LM20 and LM27 that were developed for Lasioglossum malachurum (Paxton et al. 2003) and rub02, rub04, rub06, rub30, rub35, rub37b, rub55, rub59, rub60, rub61, rub72, rub73, rub77 and rub80 that were developed for Halictus rubicundus (Soro \& Paxton 2009).

The 11 following primer pairs amplified polymorphic fragments in H. scabiosae: LHMS10, rub02, rub06, rub35, rub37b, rub55, rub59, rub60, rub72, rub 73 and rub80. These 11 loci were analysed in three multiplex polymerase chain reactions (PCR). The first PCR contained $1 \mu \mathrm{L}$ of the extraction supernatant, $0.2 \mu \mathrm{M}$ of primers rub80 and rub73, $0.1 \mu \mathrm{M}$ of primers LHMS10, $1.7 \mu \mathrm{M}$ of primers rub02, $200 \mu \mathrm{M}$ of each dNTP, 2 mM MgCl 2 , 1× QIAGEN PCR buffer, 1× QIAGEN Q solution and $0.5 \mathrm{U}$ of QIAGEN thermostable Taq DNA polymerase for a final volume of $10 \mu \mathrm{L}$. The second PCR contained $2 \mu \mathrm{L}$ of the extraction supernatant, $1.3 \mu \mathrm{M}$ of primers rub72, $3 \mu \mathrm{M}$ of primers rub06, $2.7 \mu \mathrm{M}$ of primers rub37b, $200 \mu \mathrm{M}$ of each dNTP, $2 \mathrm{~mm} \mathrm{MgCl}_{2}$, $1 \times$ QIAGEN PCR buffer, $1 \times$ QIAGEN Q solution and 0.5 U of Taq DNA polymerase for a final volume of $10 \mu \mathrm{L}$. The third PCR contained $2 \mu \mathrm{L}$ of the extraction supernatant, $2 \mu \mathrm{M}$ of primers rub35, $2.5 \mu \mathrm{M}$ of primers rub59, $1.5 \mu \mathrm{M}$ of primers rub60, $3 \mu \mathrm{M}$ of primers rub55, $200 \mu \mathrm{M}$ of each dNTP, $2 \mathrm{~mm} \mathrm{MgCl}$, $1 \times$ QIAGEN PCR buffer, $1 \times$ QIAGEN Q solution and $0.5 \mathrm{U}$ of Taq DNA polymerase for a final volume of $10 \mu \mathrm{L}$. Amplifications were carried out on GeneAmp PCR Systems 2700 or 2900 (PerkinElmer) with the following thermal profiles: initial denaturation of $2 \mathrm{~min}$ at $94{ }^{\circ} \mathrm{C}$, followed by 35 cycles (first PCR) or 45 cycles (second and third PCRs) of $45 \mathrm{~s}$ at $94{ }^{\circ} \mathrm{C}, 45$ s at $55^{\circ} \mathrm{C}$ and $1 \mathrm{~min}$ at $72{ }^{\circ} \mathrm{C}$, and a final extension of $5 \mathrm{~min}$ at $72^{\circ} \mathrm{C}$. Primers were labelled with HEX, NED and FAM fluorescent dyes (Table 1). Amplification products of the second and third PCRs were mixed in proportions $4: 3$ and analysed separately from products of the first PCR on an ABI PRISM 3100 sequencer (Applied Biosystems). Alleles were scored with GeneMapper software version 4.0. We retyped a few individuals for which multiple loci did not amplify in the first run.
Table 1 Genetic diversity of microsatellite markers in Halictus scabiosae

\begin{tabular}{llrrll}
\hline Locus & Dye & NA & \multicolumn{1}{l}{$F_{\mathrm{IS}}$} & $P$-value & $H_{\mathrm{E}}$ \\
\hline rub80 & NED & 17 & -0.019 & 0.711 & 0.74 \\
rub73 & FAM & 7 & -0.020 & 0.679 & 0.73 \\
LHMS10 & FAM & 5 & 0.182 & 0.001 & 0.63 \\
rub02 & HEX & 6 & 0.092 & 0.126 & 0.55 \\
rub06 & HEX & 23 & -0.070 & 0.996 & 0.87 \\
rub72 & HEX & 11 & -0.102 & 0.999 & 0.85 \\
rub60 & NED & 19 & -0.059 & 0.983 & 0.89 \\
rub59 & FAM & 13 & 0.007 & 0.484 & 0.82 \\
rub35 & NED & 24 & 0.002 & 0.525 & 0.90 \\
rub37b & HEX & 12 & 0.169 & 0.000 & 0.69 \\
rub55 & FAM & 35 & 0.061 & 0.032 & 0.92 \\
Overall & & 16 & 0.014 & 0.125 & 0.78 \\
\hline
\end{tabular}

The fluorescent dye used to label primers, number of alleles $\left(N_{\mathrm{A}}\right)$, Weir and Cockerham estimation of $F_{\mathrm{IS}}$ with its associated $P$ value and genetic diversity $\left(H_{\mathrm{E}}\right)$ are presented.

\section{Genetic data analysis}

Genetic diversity $H_{\mathrm{E}}$ and Weir \& Cockerham's (1984) inbreeding coefficient $F_{\mathrm{IS}}$ were estimated for each locus and over all loci using one female per nest to avoid bias due to the nonindependence of individuals from the same nest. Departure from Hardy-Weinberg expectations (i.e. the probability that $F_{\text {IS }}$ was significantly greater than zero) was tested for each locus using the randomization procedure implemented in the computer program FSTAT 2.9.4 (Goudet 1995). Linkage disequilibrium for all pairs of loci in each population was tested using the same program.

We estimated the genetic relatedness $r$ among female nestmates using the algorithm of Queller \& Goodnight (1989), as implemented in the computer program Relatedness 5.0.8. Genetic relatedness within nests was estimated relative to the total sample using allele frequencies of all males and females, weighing nests equally. Standard errors and $95 \%$ confidence intervals of $r$ were obtained by jackknifing across loci.

Specific pedigree relationships were determined by carefully examining the genotype arrays in each nest, and were further tested with the likelihood approach implemented in the computer program Kinship 1.3.1 (Goodnight \& Queller 1999). Groups of full-sisters within nests were identified by inspection of genotypes. This identification was confirmed by calculating the likelihood that pairs of females were fullsisters $(r=0.75)$ rather than half-sisters $(r=0.25$, which is more conservative than using $r=0$ ). To identify full-sisters collected in different nests, we also applied this procedure to all possible pairs of females within a population.

We inferred the genotype of queens and their mates from the genotypes in groups of sisters. These sisterhoods had been reliably identified from their multi-loci genotypes as described above. In a group of full-sisters from a haplo-diploid 
species, a maximum of three alleles is found at each locus. Indeed, the allele carried by the queen's haploid mate must be shared by all sisters, and at most two additional alleles can come from the diploid queen. The maternal origin of alleles was further checked by comparison with the haploid genotypes of male offspring, when available. The parental genotypes could not be inferred when the number of females per sisterhood was low and all females had the same homozygous genotype, or when all females had the same heterozygous genotype at a locus. We estimated the mean relatedness between queens and their mates relative to the whole sample with the program Relatedness 5.0.8. We calculated the corresponding inbreeding coefficient $F_{\text {IT }}$ with the program FSTAT 2.9.4, using one randomly selected female per nest.

Our data set comprises three hierarchical levels: individuals within nests, nests within populations and populations within the entire sample. To measure genetic differentiation between populations, we therefore applied a three-level hierarchical $F$-analysis of variance that included the individual, nest and population levels, using the computer package HierFSTAT (Goudet 2005). We tested if $F_{\mathrm{ST}}$ coefficients among populations were significantly greater than zero by permuting nests among populations. We investigated whether there was isolation by distance (a positive correlation between geographical distance and genetic differentiation among populations) with a Mantel test. For this test, we calculated $F_{\mathrm{ST}}$ between pairs of populations and tested the significance of the matrix correlation between $F_{\mathrm{ST}} /\left(1-F_{\mathrm{ST}}\right)$ and the logarithm of geographical distances with 5000 permutations of rows and columns, as implemented in the program FSTAT 2.9.4.

If dispersal is sex biased, $F_{\mathrm{ST}}$ is expected to be higher in the philopatric sex after the dispersal event (Goudet et al. 2002). We tested for sex-biased dispersal using the inferred genotypes of queens and their mates, which correspond to 'post-dispersal' females and males that successfully reproduced. Only the parental generation was included in the analysis. We estimated the $F_{\mathrm{ST}}$ for queen and father groups in separate analyses, and for each sex we tested if there was genetic differentiation among populations with 5000 randomizations, using the program FSTAT 2.9.4. To investigate whether dispersal was sex biased, we calculated the difference between male and female $F_{\mathrm{ST}}$ and tested the significance of this value by performing a one-sided test with 10000 randomizations and females set as the philopatric sex, as has been suggested from behavioural observations in several halictine species (Kukuk \& Decelles 1986; Yanega 1990; Potts \& Willmer 1998).

In male-haploid female-diploid species, a higher $F_{\mathrm{ST}}$ for females than for males conservatively indicates that dispersal is male biased. This is because a higher co-ancestry is expected among males than among females when both sexes disperse equally. Indeed, two genes sampled from two different males are necessarily inherited maternally, so there is always a non-zero probability that the two lineages coalesce in the parental generation (effective when the two males are brothers). By contrast, genes sampled from two females were inherited in only half of the cases from the same-sex parents (i.e. both maternally or both paternally), which halves the probability of coalescence in the parental generation. A higher co-ancestry among diploid females than among haploid males is therefore a strong indication of male-biased dispersal, as the reverse pattern is expected when both sexes disperse equally.

\section{Results}

\section{Field monitoring}

The monitoring of one population during the early stage of the breeding season revealed two important aspects of the nesting biology of Halictus scabiosae. First, pleometrosis (joint nest founding by multiple females) appeared to be fairly common, as 13 out of the 42 monitored nests (31\%) were occupied by two to four foundresses. This number is a minimum estimate, as some individuals might have remained undetected. Second, the beginning of the breeding season is characterized by high rates of nest failure and foundress movement. Only three out of the 42 monitored nests produced new females in summer. As many as $53 \%$ of the 99 females that we marked individually were observed only once, on the day of marking. These single captures occurred mostly in May, suggesting that foundresses move a lot or have a high mortality shortly after emerging from hibernation. It is possible that disturbance due to marking has increased the rate of nest failure. However, $42 \%$ to $73 \%$ of the nests appeared to be unoccupied in three other populations in which bees had not been marked, which further points at high failure rates during colony foundation. In the population where we marked the foundresses, $17 \%$ of the marked females were captured in more than one nest. All but one of those females were first captured before 1 June (i.e. before the emergence of new offspring). In contrast, $25 \%$ of the marked females were captured multiple times in the same nest, mostly in July.

In total, we succeeded in dissecting 394 (out of 424) females from 112 (out of 122) nests that were sampled between the end of July and September (Tables S1 and S3). Overall, 73 $(19 \%)$ of these females had active ovaries, suggesting that they had been or were close to laying eggs. We dissected several females in 66 nests $($ median $=4$, maximum $=12$ ). Out of these 66 nests, 17 nests $(26 \%)$ contained more than one female with active ovaries ( median $=2$, maximum $=5$ ).

\section{Microsatellites}

The 11 microsatellite markers were highly variable, with an average of 16 alleles and a mean expected heterozygosity 
Table 2 Sampling size and social structure for the 122 nests sampled in summer

\begin{tabular}{lll}
\hline Sample & No. of nests & Social structure \\
\hline Only one female & 52 & NA \\
Females separated by more than 12 days & 10 & NA \\
Multiple nestmates & 33 & One singly mated queen and her offspring \\
Multiple nestmates & 27 & Multiple breeders and/or drifters \\
\hline
\end{tabular}

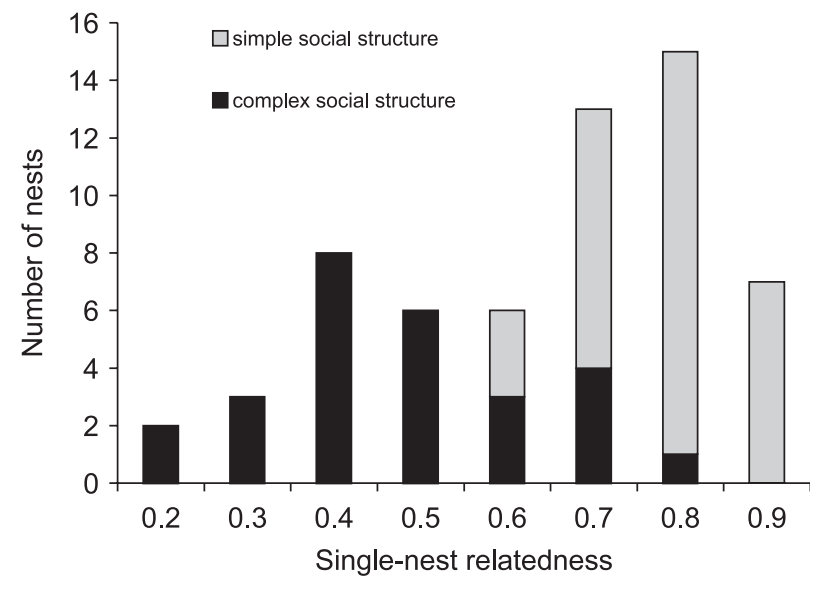

Fig. 1 Distribution of relatedness estimates among nestmate females for 60 nests of Halictus scabiosae. The social structure was inferred from the arrays of genotypes: nests with a simple social structure (grey bars) were compatible with being occupied by one singly mated queen and her daughter offspring, whereas nests with a complex social structure (black bars) had multiple breeders or drifters.

of 0.78 (Table 1). Three of the 11 microsatellites were likely to have null alleles. Indeed, rub55, rub37b and LHMS10 showed significant deviations from Hardy-Weinberg equilibrium, in sharp contrast to the eight other microsatellites (Table 1). These three markers were excluded from all population genetics analysis, but were informative for disentangling pedigree relationships and measuring within nest relatedness in the sociogenetic analyses. There was no evidence for linkage disequilibrium, except between markers rub60 and rub35 in one population.

\section{Sociogenetics}

The estimates of relatedness among female nestmates and inspection of genotype arrays revealed that the social structure varied considerably among the 60 nests for which multiple nestmate females were genotyped (Fig. 1, Table 2). In 33 of these nests (55\%), the genotype arrays were consistent with one singly mated female having produced all nestmates, with at most three alleles per locus and per nest. The occurrence of single mating is bolstered by the prevalence of monoandry in other halictine bees (reviewed in Hughes et al. 2008a). The average relatedness between females in the nests with simple families was 0.728 (95\% confidence interval 0.699-0.757; Fig. 1), which is close to the expected relatedness of 0.75 for hymenopteran full-sisters.

The remaining 27 nests (45\%) had a more complex social structure, as revealed by the presence of genotypes that could not have been produced by a single reproducing female and by the significantly lower average within-nest relatedness between females $(0.421,95 \%$ confidence interval $0.361-0.481$; Fig. 1). Four out of these 27 nests with complex social structures contained multiple groups of sisters. At least three of them were consistent with being polygynous (i.e. harboured multiple females that had reproduced simultaneously), whereas the fourth nest appeared to be functionally monogynous (see below; pedigrees and kinship analyses are provided in Table S2, Supporting information). A likelihood analysis of kinship permitted us to unambiguously delineate the groups of full sisters in these four nests. We could infer the genealogical relationships between groups of sisters from the genotype arrays and did not detect other relatives in any other nest, which together suggest that three of these four nests had multiple reproducing queens. Nest P75 contained two sisterhoods with two and four females whose genotype arrays were consistent with being the offspring of two full-sister queens that we did not sample (Table S2). Nest R27 also contained two sisterhoods with three and four females, but which had genotypes incompatible with being the offspring of two full-sisters, because the genotypes of the mothers did not share an allele at each locus (Table S2). Nest B27 harboured three sisterhoods. One of these sisterhoods had four females, of which two had active ovaries and genotypes consistent with being sisters of the mothers of the two other sisterhoods of three and two females, respectively (Table S2). The occurrence of multiple reproducing sisters was further confirmed by the presence of two and four males that had genotypes consistent with being the offspring of two females from the first sisterhood. Finally, nest B12 seemed to be functionally monogynous. It contained two groups of sisters that probably belonged to two generations, with three sister foundresses and the offspring of one of these foundresses (the queen). Specifically, five sisters and two males from this nest had genotypes consistent with being the offspring of one of the three sister foundresses that had fully active ovaries (Table S2).

A closer inspection of the genotype arrays and a likelihood analysis with the program Kinship jointly revealed 
that at least 20 out of the 122 nests analysed (and 12 out of the 27 nests with complex social structure) contained females originating from another nest in the population. In the analysis with the program Kinship, the likelihood ratio that drifters were full-sisters, as opposed to half-sisters, to bees in other nests had probabilities below 0.01 in all cases, and generally below 0.001 . These females $(n=29)$ were unrelated to their nestmates $(r=0.02,95 \% \mathrm{CI}:-0.08-0.12)$, but had genotypes consistent with being full-sisters of the females sampled in another nest ( $r=0.72,95 \%$ CI: 0.67-0.77). The vast majority of foreign bees had inactive ovaries. Specifically, of the 27 foreign bees whose degree of ovary activation could be assessed, 26 had an ovary activation score of zero, and one had a score of two. The mean distance between the nests from which the foreign bees originated and the nests in which they were sampled was only $23.2 \mathrm{~cm}$ ( \pm SE $3.9 \mathrm{~cm}, n=22$ ), which contrasts with the mean internest distance of $360 \mathrm{~cm}( \pm$ SE $14 \mathrm{~cm}, n=1138)$ within the same populations. The nests from which foreign bees originated contained significantly more females than average in the study population (mean $=7.1, n=17$ nests, versus 3.9 females per nest, $n=121$, respectively; two-sided Mann-Whitney test: $P<0.001)$. All drifters were sampled after 15 August.

The analysis of genotypes of sisterhoods allowed us to reconstruct the genotypes of 44 queens and 46 of their mates. The mean relatedness between queens and their mates was low $(r=0.076,95 \%$ CI: $-0.049-0.200)$. In line with this finding, the inbreeding coefficient $F_{\mathrm{IT}}$ calculated with one random bee per nest indicated that mating occurred mainly between unrelated individuals (mean $\pm \mathrm{SE}=-0.013 \pm 0.013,95 \%$ $\mathrm{CI}=-0.036-0.013, P=0.82)$.

\section{Population genetics and sex-biased dispersal}

Genetic differentiation among populations was weak but significantly greater than zero $\left(F_{\mathrm{ST}}=0.02, P=0.01\right)$. Half of the pairwise population comparisons yielded significant $F_{\mathrm{ST}}$ values (Table 3 ). Two populations, $\mathrm{S}$ and J, showed significant genetic differentiation with most of the other populations.

Genetic differentiation between pairs of populations was not significantly correlated with geographical distance (correlation $=0.21, P=0.28$ ). This is in part because population $S$, although not geographically isolated, had high pairwise $F_{\text {ST }}$ values compared to other populations. Population $S$ was small and had low gene diversity $(0.65 \pm 0.05)$ relative to the mean of other populations $(0.79 \pm 0.02)$, which is consistent with a founder effect associated with a recent colonization event.

The $F_{\mathrm{ST}}$ for the reproductive queens based on inferred genotypes from sisterhoods (mean $\pm \mathrm{SE}=0.034 \pm 0.011,95 \%$ $\mathrm{CI}=0.018-0.060, P<0.001)$ was greater than that for their male mates $(-0.001 \pm 0.010,95 \% \mathrm{CI}=-0.018-0.019$, NS), and
Table 3 Pairwise $F_{\mathrm{ST}}$ values between populations and their significance

\begin{tabular}{lrlllllll}
\hline & $\mathrm{B}$ & $\mathrm{C}$ & $\mathrm{H}$ & $\mathrm{J}$ & $\mathrm{P}$ & $\mathrm{R}$ & $\mathrm{S}$ & $\mathrm{T}$ \\
\hline $\mathrm{B}$ & & $\mathrm{NS}$ & $\mathrm{NS}$ & $\mathrm{NS}$ & $* *$ & $* *$ & $\mathrm{NS}$ & $\mathrm{NS}$ \\
$\mathrm{C}$ & -0.009 & & $\mathrm{NS}$ & $* *$ & $\mathrm{NS}$ & $\mathrm{NS}$ & $* *$ & $*$ \\
$\mathrm{H}$ & -0.006 & 0.000 & & $*$ & $\mathrm{NS}$ & $\mathrm{NS}$ & $* *$ & $\mathrm{NS}$ \\
$\mathrm{J}$ & 0.012 & 0.015 & 0.023 & & $* *$ & $*$ & $* *$ & $* *$ \\
$\mathrm{P}$ & 0.014 & 0.015 & 0.013 & 0.044 & & $*$ & $* *$ & $\mathrm{NS}$ \\
$\mathrm{R}$ & 0.013 & 0.013 & 0.017 & 0.043 & 0.007 & & $* *$ & $\mathrm{NS}$ \\
$\mathrm{S}$ & 0.038 & 0.052 & 0.049 & 0.081 & 0.063 & 0.083 & & $\mathrm{NS}$ \\
$\mathrm{T}$ & 0.012 & 0.011 & 0.001 & 0.025 & 0.018 & 0.036 & -0.007 & \\
\hline
\end{tabular}

Above diagonal, $*: P<0.05, * *: P<0.01$.

the test for male-biased dispersal was close to being significant $(P=0.078)$. This test is however conservative in haplodiploid species (see material and methods). Hence, the fact that we found a higher co-ancestry among diploid females than among haploid males, whereas the reverse pattern is expected when both sexes disperse equally, is consistent with male-biased dispersal in our study populations.

\section{Discussion}

Sweat bees are model systems to study the evolution of sociality, because females have flexible reproductive strategies and often exhibit facultative helping behaviour (Schwarz et al. 2007). Our fine-scale microsatellite analysis coupled with field observations indeed revealed that females of Halictus scabiosae exhibit a great behavioural flexibility throughout their life cycle, which results in highly variable social organization, from simple families with one female helped by her daughters to groups of females with variable levels of relatedness and reproductive shares.

In spring, females founded nests either alone or in association with other females. Pleometrosis appeared to be common, as we observed two to four females in about one third of the nests during the beginning of the breeding season. In contrast, our genetic analysis revealed that a single female actually produced adult offspring in most of the nests, and the sharing of reproduction by multiple females was detected in only three out of 60 nests (5\%). The most likely explanation for this pattern is that one of the foundresses usually becomes reproductively dominant. Dominance behaviour has indeed been reported among co-foundresses of $H$. scabiosae, with the dominant female expelling subordinates from the nest before offspring emergence (Knerer \& Plateaux-Quénu 1966, 1967). This unequal partitioning of reproduction results in a prevalence of nests with simple families and high relatedness in $H$. scabiosae.

Genetic studies have documented monogyny and monoandry for several halictine bee species (Crozier et al. 1987; Mueller et al. 1994; Richards et al. 2005), sometimes coupled 
with queen replacement by a daughter in cases of orphaning (Mueller 1991; Packer \& Owen 1994). In contrast, polygyny and facultative polyandry appear to be rare, the latter having been documented in only one species (Richards et al. 1995). Overall, the rarity of polygyny and polyandry in halictine bees supports the hypothesis that they are derived traits and that kin selection is central to the evolution of eusociality in this group (Hughes et al. 2008a, b).

The genetic data in H. scabiosae show that co-foundresses rarely get a share of reproduction in the nest they helped to establish. Why, then, do they not reproduce solitarily? The answer depends on the inclusive fitness benefits of subordinates, ecological constraints on solitary breeding, and probability that a co-foundress will become the dominant egg-layer (Packer 1986; Keller \& Reeve 1994; Tibbetts \& Reeve 2003; Field et al. 2006). There is some indirect evidence that in H. scabiosae, subordinate co-foundresses may increase their inclusive fitness by helping the reproducing queen. First, co-foundresses are likely to be highly related, because sisters often hibernate together in the natal nest (PlateauxQuénu 1972), and we indeed found sister foundresses in our study population. Females of Halictus ligatus hibernate in tunnels under the natal nest and also exhibit a moderate proportion of pleometrotic nests (Packer 1986; Richards \& Packer 1998). Second, the high rate of nest failure observed in our field population suggests that ecological constraints are high during the nest founding stage. Pleometrosis is likely to increase the probability of success of the nest during that crucial stage, as it improves nest guarding and reduces cleptoparasitism in halictine bees (Lin 1964; Abrams \& Eickwort 1981). Indeed, pleometrotic nests had a higher survival than haplometrotic ones in H. ligatus (Richards \& Packer 1998) and social nests had lower failure rate and higher productivity than solitary ones in Megalopta genalis (Smith et al. 2007). Finally, co-foundresses might also have a chance to become the dominant egg-layer (Field et al. 2006), and in that case may even be unrelated, as occurs in the paper wasp Polistes dominulus (Queller et al. 2000; Zanette \& Field 2008). In our survey of $H$. scabiosae, at least one unrelated female had active ovaries and one nest contained two sisterhoods whose mothers could not be sisters.

A surprising finding of our study is that we detected females originating from foreign nests in $16 \%$ of the overall sample of nests, and in $44 \%$ of the nests with complex social structure. These bees were unrelated to their nestmates but were full-sisters of individuals in another nest. These estimates are minimal values, as more foreign bees might have originated from nests that had not been sampled. Two explanations may account for this pattern. First, queens may have laid eggs in neighbouring nests. Second, adult bees might have drifted to neighbouring nests. The second explanation is more likely, because in eight out of the 20 nests containing foreign bees, only foreigners were sampled, which suggests that they had drifted to unoccupied nests.
Moreover, the foreigners came from nests with aboveaverage productivity and they were all caught late in the breeding season, after 15 August.

Drifting was recently found to be relatively common in honeybees (Nanork et al. 2005, 2007), bumblebees (Birmingham et al. 2004; Lopez-Vaamonde et al. 2004) and Polistes wasps (Sumner et al. 2007), and might have been largely overlooked in the social insects so far (reviewed by Beekman \& Oldroyd 2008). Radio-tagging revealed a very high rate of drifting in Polistes canadensis. In this species, drifters moved mostly towards nests containing closely related individuals, which suggests that drifters increase their inclusive fitness (Sumner et al. 2007). In contrast, drifting in bumblebees and honeybees were associated with increased reproduction of drifters in non-natal nests, which indicates that drifting is a form of social parasitism (Birmingham et al. 2004; LopezVaamonde et al. 2004; Nanork et al. 2005, 2007). In halictine bees, microsatellite analyses have shown that unrelated workers are occasionally present in Lasioglossum malachurum nests (Paxton et al. 2002; Richards et al. 2005) and females of H. scabiosae occasionally took over nests of another species (Knerer \& Plateaux-Quénu 1967).

Drifting in H. scabiosae seems unlikely to increase the indirect component of the inclusive fitness of drifters, for two reasons. First, drifters were unrelated to the members of their host nests. Second, drifters were found late in the breeding season, at a time when host nests did not rear new brood. The second point also makes it unlikely that drifters had reproduced in their host nest, which was confirmed by the lack of ovary activity for the vast majority of drifters.

Our data suggest that drifting might be a way to decrease reproductive competition among related females that are about to enter hibernation. If a female comes from a productive nest, overwintering in the natal nest with many sisters is associated with a high probability of being expelled from the nest by a dominant sister during the next spring. In contrast, drifting to an empty or less populated nest before hibernation, alone or with a few sisters, is likely to increase the chances of direct reproduction in the next breeding season. The fact that drifters came mostly from productive nests and that we captured only drifters in four nests is consistent with this hypothesis. A similar pattern of female dispersal has been documented in the halictine bee Lasioglossum (Chilalictus) hemichalceum. Nest re-use is frequent in this species (Kukuk 2002) and interestingly, part of the females left their natal nests before hibernation, particularly when nests were large (Kukuk et al. 2005).

In addition to frequent short-range dispersal by drifting, our genetic data provide evidence for random mating and long-range dispersal in H. scabiosae. Two lines of evidence indicate that mating occurs at random in our study populations. First, queens were unrelated to their mates. Second, the inbreeding coefficient of females was indistinguishable 
from zero. Across populations, the average genetic differentiation was small but significantly greater than zero, amounting to a global $F_{\mathrm{ST}}$ of $2 \%$. The pairwise $F_{\mathrm{ST}}$ was significant for mainly two populations that appeared to be genetically differentiated from the others. One population (J) was geographically and ecologically isolated, being in the city $3.5 \mathrm{~km}$ away from the campus (Fig. S1). For the other population (S), the genetic differentiation might be due to the recent colonization of the patch by a small number of founders. We sampled adjacent as well as more distant populations (Fig. S1), which should have permitted to detect a strong pattern of isolation by distance over the scale investigated. The overall lack of isolation by distance among populations therefore suggests that some males and/or females disperse over long distances. Hence, these data on mating system and population differentiation suggest fairly extensive gene flow within and between populations, even if some newly founded populations may become temporarily differentiated.

The comparison of the degree of genetic differentiation in females and males sampled after dispersal indicates that males disperse more than females in H. scabiosae. This is in line with observations from several authors, who have suggested that females are philopatric in sweat bees, as they tend to re-use or stay close to their natal nests (Kukuk \& Decelles 1986; Yanega 1990; Potts \& Willmer 1998), while males have been shown to prefer females from a population other than their own in Lasioglossum zephyrum and L. malachurum (Smith \& Ayasse 1987). It is also consistent with the hypothesis that short-distance drifting might decrease local resource competition among related females. But even if dispersal is globally male biased, our field observations indicate that females are far from being completely philopatric. Extensive female movement occurred during the nest-founding stage, with a decrease in the number of active nests, the disappearance of many individuals, and occasional movements to neighbouring nests, up to $20 \mathrm{~m}$ away. Moreover, some long-distance dispersal by females is also expected from the ecology of $H$. scabiosae and other groundnesting bees that are pioneer species occupying unstable, anthropogenic and patchy habitat with low vegetation cover (Packer \& Owen 2001).

Altogether, the microsatellite data revealed an unusually high level of behavioural plasticity in H. scabiosae throughout its life cycle, which resulted in highly variable social structure and alternative dispersal strategies, with malebiased dispersal overall and occasional colonization of distant patches by females, as well as frequent drifting of females from one nest to the other within populations. This diversity calls for further studies on the ecological and social correlates of plastic behaviour, and in particular on the adaptive nature of joint nest founding, drifting and patch colonization.

\section{Acknowledgements}

We thank Nadège Remollino for help in the laboratory, Robert J. Paxton for providing us with microsatellite primer sequences ahead of publication, Andrew F.G. Bourke, Jérôme Goudet, Jean-François Huertas, Morgan Pearcy and Robert J. Paxton for discussion or comments on the manuscript. This research was supported by grant 3100AO-108263 from the Swiss National Science Foundation.

\section{References}

Abrams J, Eickwort GC (1981) Nest switching and guarding by the communal sweat bee Agapostemon virescens (Hymenoptera, Halictidae). Insectes Sociaux, 28, 105-116.

Batra SWT (1966) Nesting behavior of Halictus scabiosae in Switzerland (Hymenoptera Halictidae). Insectes Sociaux, 13, 87-92.

Beekman M, Oldroyd BP (2008) When workers disunite: intraspecific parasitism by eusocial bees. Annual Review of Entomology, 53, 19-37.

Beekman M, Peeters C, O'Riain MJ (2006) Developmental divergence: neglected variable in understanding the evolution of reproductive skew in social animals. Behavioral Ecology, 17, 622627.

Birmingham AL, Hoover SE, Winston ML, Ydenberg RC (2004) Drifting bumble bee (Hymenoptera: Apidae) workers in commercial greenhouses may be social parasites. Canadian Journal of Zoology, 82, 1843-1853.

Brady SG, Sipes S, Pearson A, Danforth BN (2006) Recent and simultaneous origins of eusociality in halictid bees. Proceedings of the Royal Society B: Biological Sciences, 273, 1643-1649.

Chapuisat M, Bocherens S, Rosset H (2004) Variable queen number in ant colonies: no impact on queen turnover, inbreeding, and population genetic differentiation in the ant Formica selysi. Evolution, 58, 1064-1072.

Clutton-Brock T (2002) Breeding together: kin selection and mutualism in cooperative vertebrates. Science, 296, 69-72.

Crozier RH, Smith BH, Crozier YC (1987) Relatedness and population structure of the primitively eusocial bee Lasioglossum zephyrum (Hymenoptera: Halictidae) in Kansas. Evolution, 41, 902-910.

Danforth BN (2002) Evolution of sociality in a primitively eusocial lineage of bees. Proceedings of the National Academy of Sciences, USA, 99, 286-290.

Eickwort GC, Eickwort JM, Gordon J, Eickwort MA (1996) Solitary behavior in a high altitude population of the social sweat bee Halictus rubicundus (Hymenoptera: Halictidae). Behavioral Ecology and Sociobiology, 38, 227-233.

Field J, Cronin A, Bridge C (2006) Future fitness and helping in social queues. Nature, 441, 214-217.

Goodnight KF, Queller DC (1999) Computer software for performing likelihood tests of pedigree relationship using genetic markers. Molecular Ecology, 8, 1231-1234.

Goudet J (1995) FSTAT (version 1.2): a computer program to calculate F-statisics. Journal of Heredity, 86, 485-486.

Goudet J (2005) Hierfstat, a package for R to compute and test hierarchical F-statistics. Molecular Ecology Notes, 5, 184-186.

Goudet J, Perrin N, Waser P (2002) Tests for sex-biased dispersal using bi-parentally inherited genetic markers. Molecular Ecology, 11, 1103-1114.

Griffin AS, West SA (2003) Kin discrimination and the benefit of helping in cooperatively breeding vertebrates. Science, 302, 634-636. 
Hart AG, Ratnieks FLW (2005) Crossing the taxonomic divide: conflict and its resolution in societies of reproductively totipotent individuals. Journal of Evolutionary Biology, 18, 383395.

Hughes WOH, Oldroyd BP, Beekman M, Ratnieks FLW (2008a) Ancestral monogamy shows kin selection is key to the evolution of eusociality. Science, 320, 1213-1216.

Hughes WOH, Ratnieks FLW, Oldroyd BP (2008b) Multiple paternity or multiple queens: two routes to greater intracolonial genetic diversity in the eusocial Hymenoptera. Journal of Evolutionary Biology, 21, 1090-1095.

Keller L, Reeve HK (1994) Partitioning of reproduction in animal societies. Trends in Ecology E Evolution, 9, 98-102.

Knerer G, Plateaux-Quénu C (1966) Sur la polygynie chez les halictinae (Insectes Hyménoptères). Comptes Rendus de l'Académie des Sciences (Paris), 263, 2014-2017.

Knerer G, Plateaux-Quénu C (1967) Usurpation de nids étrangers et parasitisme facultatif chez Halictus scabiosae (Rossi) (Insecte Hyménoptère). Insectes Sociaux, 14, 47-50.

Kukuk PF (2002) Nest reuse in a communal halictine bee, Lasioglossum (Chilalictus) hemichalceum (Hymenoptera: Halictidae). Journal of the Kansas Entomological Society, 75, 3-7.

Kukuk PF, Decelles PC (1986) Behavioral evidence for population structure in Lasioglossum (Dialictus) zephyrum: female dispersion patterns. Behavioral Ecology and Sociobiology, 19, 233-239.

Kukuk PF, Forbes SH, Zahorchack R, Riddle A, Pilgrim K (2002) Highly polymorphic microsatellite markers developed for the social halictine bee Lasioglossum (Chilalictus) hemichalceum. Molecular Ecology Notes, 2, 529-530.

Kukuk PF, Bitney C, Forbes SH (2005) Maintaining low intragroup relatedness: evolutionary stability of nonkin social groups. Animal Behaviour, 70, 1305-1311.

Lin N (1964) Increased parasitic pressure as a major factor in the evolution of social behavior in halictine bees. Insectes Sociaux, 11, 187-192.

Lopez-Vaamonde C, Koning JW, Brown RM, Jordan WC, Bourke AFG (2004) Social parasitism by male-producing reproductive workers in a eusocial insect. Nature, 430, 557-560.

Mueller UG (1991) Haplodipoidy and the evolution of facultative sex ratios in a primitively eusocial bee. Science, 254, 442-444.

Mueller UG, Eickwort GC, Aquadro CF (1994) DNA fingerprinting analysis of parent-offspring conflict in a bee. Proceedings of the National Academy of Sciences, USA, 91, 5143-5147.

Nanork P, Paar J, Chapman NC, Wongsiri S, Oldroyd BP (2005) Asian honeybees parasitize the future dead. Nature, 437, 829829.

Nanork P, Chapman NC, Wongsiri S et al. (2007) Social parasitism by workers in queenless and queenright Apis cerana colonies. Molecular Ecology, 16, 1107-1114.

Packer L (1986) Multiple-foundress associations in a temperate population of Halictus ligatus (Hymenoptera, Halictidae). Canadian Journal of Zoology, 64, 2325-2332.

Packer L, Owen RE (1994) Relatedness and sex ratio in a primitively eusocial halictine bee. Behavioral Ecology and Sociobiology, 34, 1-10.

Packer L, Owen R (2001) Population genetic aspects of pollinator decline. Conservation Ecology, 5, art. 4, Available from URL: http://www.consecol.org/vol5/iss1/art4.

Paxton RJ, Ayasse M, Field J, Soro A (2002) Complex sociogenetic organization and reproductive skew in a primitively eusocial sweat bee, Lasioglossum malachurum, as revealed by microsatellites. Molecular Ecology, 11, 2405-2416.
Paxton RJ, Arevalo E, Field J (2003) Microsatellite loci for the eusocial Lasioglossum malachurum and other sweat bees (Hymenoptera, Halictidae). Molecular Ecology Notes, 3, 82-84.

Plateaux-Quénu C (1972) La Biologie des Abeilles Primitives. Masson $\&$ Cie, Paris.

Potts SG, Willmer P (1998) Compact housing in built-up areas: spatial patterning of nests in aggregations of a ground-nesting bee. Ecological Entomology, 23, 427-432.

Queller DC, Goodnight KF (1989) Estimating relatedness using genetic markers. Evolution, 242, 258-275.

Queller DC, Zacchi F, Cervo R et al. (2000) Unrelated helpers in a social insect. Nature, 405, 784-787.

Richards MH (2000) Evidence for geographic variation in colony social organization in an obligately social sweat bee, Lasioglossum malachurum Kirby (Hymenoptera; Halictidae). Canadian Journal of Zoology, 78, 1259-1266.

Richards MH, Packer L (1998) Demography and relatedness in multiple-foundress nests of the social sweat bee, Halictus ligatus. Insectes Sociaux, 45, 97-109.

Richards MH, Packer L, Seger J (1995) Unexpected patterns of parentage and relatedness in a primitively eusocial bee. Nature, 373, 239-241.

Richards MH, von Wettberg EJ, Rutgers AC (2003) A novel social polymorphism in a primitively eusocial bee. Proceedings of the National Academy of Sciences, USA, 100, 7175-7180.

Richards MH, French D, Paxton RJ (2005) It's good to be queen: classically eusocial colony structure and low worker fitness in an obligately social sweat bee. Molecular Ecology, 14, 4123-4133.

Ross KG (2001) Molecular ecology of social behaviour: analyses of breeding systems and genetic structure. Molecular Ecology, 10, 265-284.

Schwarz MP, Richards MH, Danforth BN (2007) Changing paradigms in insect social evolution: Insights from halictine and allodapine bees. Annual Review of Entomology, 52, 127-150.

Smith BH, Ayasse M (1987) Kin-based male mating preferences in two species of halictine bee. Behavioral Ecology and Sociobiology, 20, 313-318.

Smith AR, Wcislo WT, O'Donnell S (2007) Survival and productivity benefits to social nesting in the sweat bee Megalopta genalis (Hymenoptera: Halictidae). Behavioral Ecology and Sociobiology, 61, 1111-1120.

Soro A, Paxton RJ (2009) Characterization of 14 polymorphic microsatellite loci for the facultatively eusocial sweat bee Halictus rubicundus (Hymenoptera, Halictidae) and their variability in related species. Molecular Ecology Resources, 9, 150152.

Sumner S, Lucas E, Barker J, Isaac N (2007) Radio-tagging technology reveals extreme nest-drifting behavior in a eusocial insect. Current Biology, 17, 140-145.

Tibbetts EA, Reeve HK (2003) Benefits of foundress associations in the paper wasp Polistes dominulus: increased productivity and survival, but no assurance of fitness returns. Behavioral Ecology, 14, 510-514.

Weir BS, Cockerham CC (1984) Estimating F-statistics for the analysis of population structure. Evolution, 38, 1358-1370.

Yanega D (1988) Social plasticity and early-diapausing females in a primitively social bee. Proceedings of the National Academy of Sciences, USA, 85, 4374-4377.

Yanega D (1989) Caste determination and differential diapause within the first brood of Halictus rubicundus. New York (Hymenoptera, Halictidae). Behavioral Ecology and Sociobiology, 24, 97-107. 
Yanega D (1990) Philopatry and nest founding in a primitively social bee, Halictus rubicundus. Behavioral Ecology and Sociobiology, $27,37-42$.

Zanette LRS, Field J (2008) Genetic relatedness in early associations of Polistes dominulus: from related to unrelated helpers. Molecular Ecology, 17, 2590-2597.

This work was part of the Master thesis of Yuko Ulrich. Yuko is currently pursuing a $\mathrm{PhD}$ on the genomics and ecology of bumblebee immune defences at the ETH Zürich. Nicolas Perrin works on dispersal, metapopulation dynamics and genetics, focussing on sex-specific patterns in various species such as shrews and tree frogs. Michel Chapuisat studies the evolution of social behaviour in ants and bees, with a focus on kin conflicts, dispersal, social structure variation and defences against parasites.

\section{Supporting information}

Additional supporting information may be found in the online version of this article:
Fig. S1 Map of the study site, with the eight Halictus scabiosae populations.

Table S1 Location of the study populations, sampling dates and sample sizes

Table S2 Pedigrees and kinship analyses for the four nests containing multiple groups of sisters

Table S3 Complete census information for all females (F) and males (M) captured in each nest and population (Pop)

Please note: Wiley-Blackwell are not responsible for the content or functionality of any supporting materials supplied by the authors. Any queries (other than missing material) should be directed to the corresponding author for the article. 